\title{
Measuring the small-signal response of diode rectifier loads using a DSP controlled switching converter system.
}

\author{
H.D. Laird, Member, IEEE, S.D. Round, Senior Member, IEEE, R.M. Duke, Member, IEEE
}

\begin{abstract}
The assumption that non-linear rectifier loads contribute only to the steady state of a power system is commonly made in the design and analysis of power conditioners such as the shunt active filter. This tacitly assumes that the non-linear rectifier load is less sensitive than the AC system. This paper presents experimental measurements of the sensitivity of non-linear rectifiers made by injecting a perturbing current into the point of coupling of the rectifier. The perturbing current is generated with a DSP controlled switching power converter typically used in shunt active filters. The DSP system also measures the change in the load current. Results are presented for both single-phase and three-phase rectifiers. These show that both these rectifiers are sensitive to the perturbing current, that the response is measurable with relatively unsophisticated hardware and that the response has a well defined pattern in the frequencies that occur.
\end{abstract}

Keywords - Rectifier, small-signal, shunt active filter, power conditioning, measurements

\section{INTRODUCTION}

The loads connected at the customer end of the electrical supply network generally operate without significant problems. For this reason the character and behavior of these loads is not well known. However the unknown nature of the loads are of concern as the number of non-linear rectifiers increases [1].

One area of present consideration in end user power systems is the use of power conditioners to prevent any harmful effects from rectifier loads entering the power system and also to prevent harm being delivered to the load by the power system. The present thinking on power system conditioning assumes that devices that return or move the voltages and currents back to their sinusoidal ideals improve power quality. This view of power quality is simplistic with perhaps a better approach being putting an economic value on the quality of the delivered electric power. The main obstacle to this is determining what value power quality has for the customer. Survey methods attempt to quantify costs from customer's own estimates of the cost of power quality events. This however does not quantitatively asses the sensitivity of the customer's load and hence the effectiveness of any installed power conditioner. This load sensitivity is an area of research that has received little attention. However as the number of installed rectifier non-linear loads and power conditioners increase it is im-

H.D. Laird, S. D Round and R. M. Duke are with the Dept of Electrical and Computer Engineering, University of Canterbury, Private Bag 4800, Christchurch, New Zealand

$\begin{array}{ccc}\text { Corresponding Author: Hamish } & \text { Laird } \\ \text { h.laird@elec.canterbury.ac.nz } & \end{array}$ portant to determine the behavior of the loads to ensure that the power delivery system continues to operate correctly. Active power conditioners such as the shunt active filter typically have a power converter. This is switched to create a current waveform to compensate for the unwanted components of the voltage or current. By adding to the control of the power converter the ability to inject an arbitrary perturbing current, the conditioner can be made into an active measurement device for the combined $\mathrm{AC}$ system/load network to which it is connected.

This paper presents experimental results measured using an actual SAF system as a perturbing source to perform measurements of the small-signal behavior and sensitivity of a single-phase and a three phase rectifier. These measurements have been confirmed with analytic models and simulation by the authors [2].

\section{The SMall-signal Response of a ReCtifier LOAD}

Measurements are made of the small signal behavior of the load. The base or unperturbed operation of the load is driven by the fundamental $\mathrm{AC}$ voltage while the response to a small superimposed perturbing signal is termed the small signal response. This separation of the response into the unperturbed or base case and the incremental or small signal change is typical in the analysis of non-linear switching systems [3]. It is typical to assume that the base case or operating point is constant.

In an uncontrolled diode rectifier the fundamental system voltage drives the switching of by alternately forward biasing different pairs of diodes. If the devices are controlled turn on devices (thyristors) the fundamental frequency drives the switching but the turn on switching instants are controlled. In both these cases the small-signal behavior of the rectifier is determined by the interaction between the fundamental voltage driven switching and the perturbing signal.

A rectifier's essential function is to act as a modulator converting the fundamental $50 \mathrm{~Hz} \mathrm{AC}$ side voltage to $0 \mathrm{~Hz}$ DC side voltage. As part of this function other current and voltage harmonics occur. When a non-harmonic frequency is applied to the rectifier the response is at a number of frequencies algebraically related to the original applied. One way to represent this is to view the rectifier in the frequency domain and represent it with a frequency transfer matrix. This matrix has a banded structure where each non-zero element represents a modulation from one frequency to another allowing the frequency changing nature of modula- 
the transfer. The transfer from the input, $I_{I n j}$, to the measured change in the output load current $\Delta I_{L}$ is given by (1) where $\mathbf{I}$ is the identity matrix.

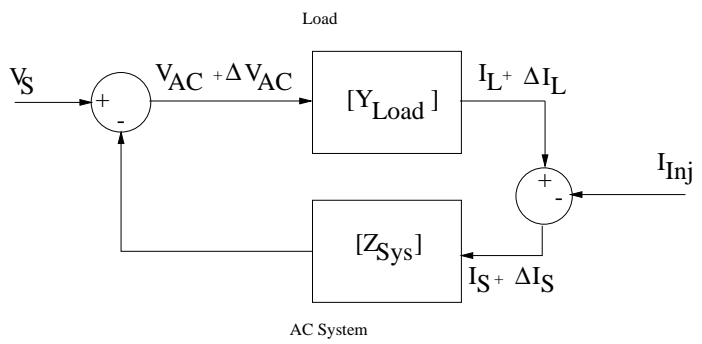

Fig. 3. Experimental system block diagram showing injected current Iinj and the resulting changes marked with $\Delta$.

$$
\Delta I_{L}=\left[\mathbf{Y}_{\mathbf{L o a d}}\right]\left[\mathbf{Z}_{\mathbf{S y s}}\right]\left[\mathbf{I}+\left[\mathbf{Y}_{\mathbf{L o a d}}\right]\left[\mathbf{Z}_{\mathbf{S y s}}\right]\right]^{-1} I_{I n j}
$$

This control block diagram shows how the response of the load cannot be separated from that of the $\mathrm{AC}$ system and demonstrates that the measurement is of the combination of the load and the AC system rather than only the load. The block diagram also serves to illustrate that the transfer from the injected current to the load current is the forward transfer of a load measurement SAF and so has control implications for the shunt active filter. If the load presents a capacitive impedance its interaction with the typically inductive system impedance causes a current measurement SAF to be unstable [6]. Showing these two parts in a block diagram form gives important insight into how the rectifier and the system interact.

\section{Single-Phase RECTIFIER MEASUREMEnts}

The experimental system is set up with the single-phase rectifier load connected line to line. Fig. 4 shows the experimentally measured base case current waveform of the single-phase diode rectifier. This has the typical peaked waveform that is usually associated with diode rectifiers. Also shown is the spectrum of this current which has the expected odd harmonic content. The converter system is
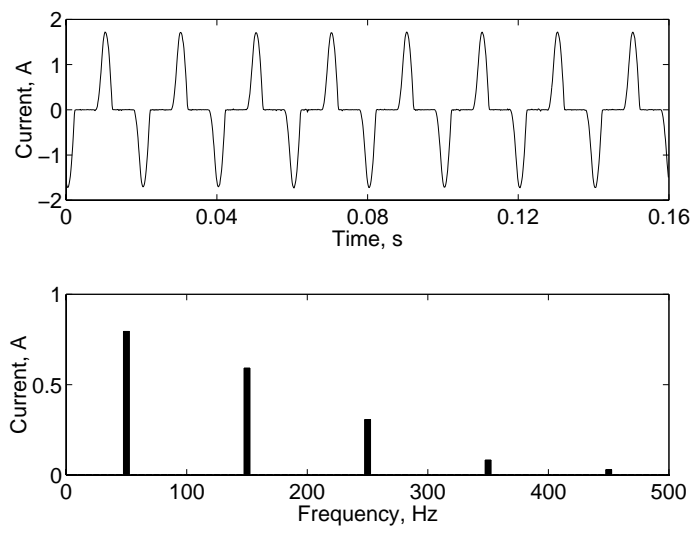

Fig. 4. Base unperturbed rectifier current waveform (Upper) and spectrum (Lower). made to generate a single frequency constant amplitude current injections. Fig. 5 shows the effect of injecting the sinusoidal perturbing current $I_{I n j}$ of $0.365 \mathrm{~A}$ (peak) at frequency of $18.75 \mathrm{~Hz}$ on the current flowing into the singlephase diode rectifier load. As can be seen by close examination of the rectifier current there is a oscillatory variation in the load current waveform away from its base case. The spectrum of the perturbed load current wave-

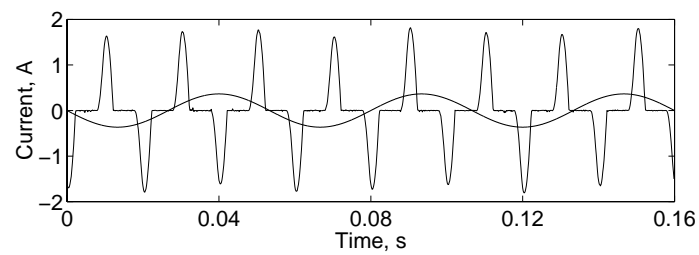

Fig. 5. Perturbed rectifier load current, $I_{L}+\Delta I_{L}$, waveform for perturbing current $I_{I n j}$ of $0.365 \mathrm{~A}$ (peak) at frequency of $18.75 \mathrm{~Hz}$.

form, $I_{L}+\Delta I_{L}$, is shown in Fig. 6 (Upper). Removing the base case, $I_{L}$, spectrum leaves the spectrum of the only the small-signal response, $\Delta I_{L}$, as is shown in Fig. 6 (Lower). Notice that the spectrum of $\Delta I_{L}$ has components at frequencies $18.75 \mathrm{~Hz}, 100-18.75=81.25 \mathrm{~Hz}$, $100+18.75=118.75 \mathrm{~Hz}$ and that this pattern continues. This shows the frequency coupling behavior of the singlephase rectifier and that it has a small-signal response characterized by a one frequency perturbation to a many frequency response [2]. Another example of single-phase rec-
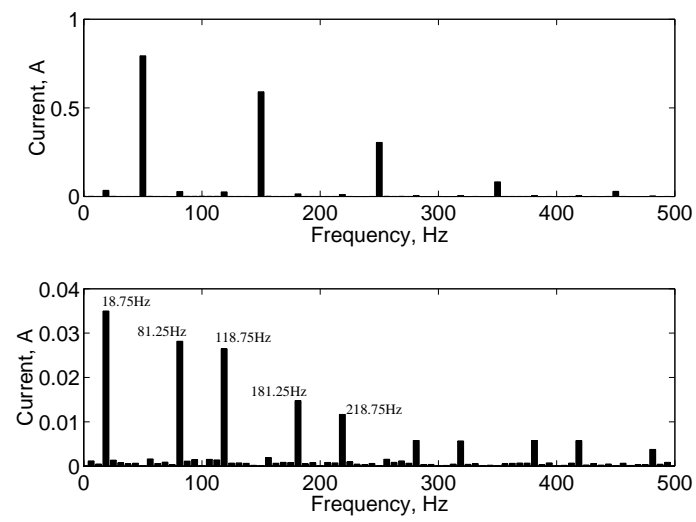

Fig. 6. Perturbed rectifier load current spectrum, $I_{L}+\Delta I_{L}$, for perturbing current $I_{I n j}$ of $0.365 \mathrm{~A}$ (peak) at frequency of $18.75 \mathrm{~Hz}$. Upper includes base case. Lower has base case removed

tifier behavior is shown in Fig. 7(a). This shows the total waveform of the load current $I_{L}+\Delta I_{L}$ and the spectra of the $\Delta I_{L}$. Notice, as previously, the variation of the current waveform. Also note that once again the resulting frequencies have the same pattern as before in that components occur at the same frequency as the applied perturbing current $62.5 \mathrm{~Hz}$, at $100-62.5=37.5 \mathrm{~Hz}$ and at $100+62.5=162.5 \mathrm{~Hz}$. Fig. 7(b) shows the effect of injecting a $318.75 \mathrm{~Hz}$ perturbing current. The amplitude of the response is smaller but the spectrum conforms to the same pattern as the previous two examples.

The frequency coupling pattern of the single-phase rec- 

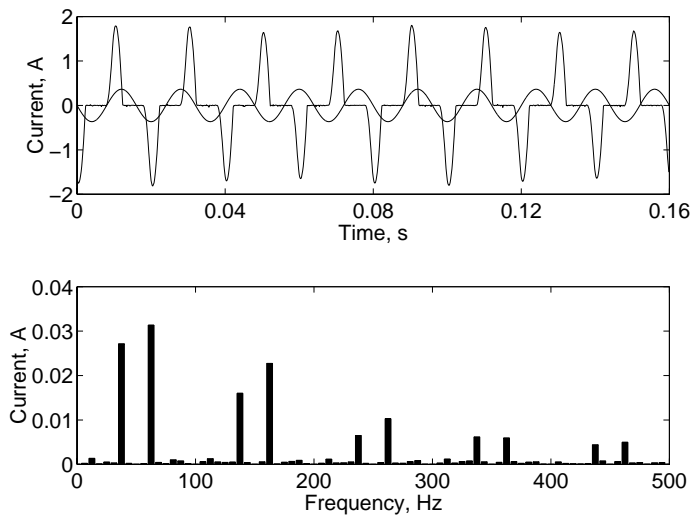

(a) Perturbing frequency $62.5 \mathrm{~Hz}$
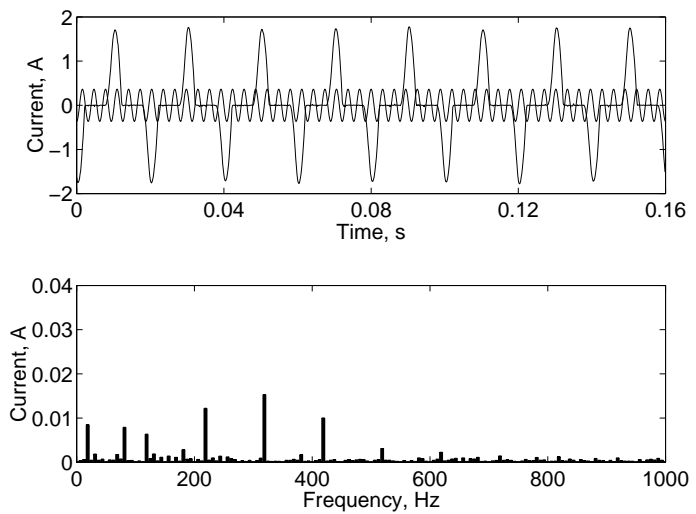

(b) Perturbing frequency $318.75 \mathrm{~Hz}$

Fig. 7. Single-phase rectifier load current waveforms and spectra for other perturbing current frequencies.

tifier is shown in Fig. 8. This shows frequency components that arise in the output load current for a given input frequency and is measured by taking FFTs of the load current for increasing injected perturbing current frequencies. The base case harmonic currents, which form the horizontal lines, are retained. This shows the way in which a perturbing current at a given frequency gives rise to a number of frequencies in the output. Consider an applied $25 \mathrm{~Hz}$ signal. By tracing down the imaginary vertical line from $25 \mathrm{~Hz}$ the frequencies that arise can be found. Ignoring the characteristic harmonics these are $25 \mathrm{~Hz}, 100-25=75 \mathrm{~Hz}$, $100+25=125 \mathrm{~Hz}, 175 \mathrm{~Hz}, 225 \mathrm{~Hz} \ldots$. The diagonal lines show that the component frequencies in the load current have a first order relation to the applied perturbing frequency. That is the output has frequencies that are related only to some arbitrary frequency plus or minus the input frequency and not to twice or some other multiple of the input frequency. This has important implications for modeling and analysis of rectifier devices [7]. The disappearance of some of the diagonal lines as the input perturbing frequency increases is caused by the magnitude of the resulting component falling below the plotting cutoff

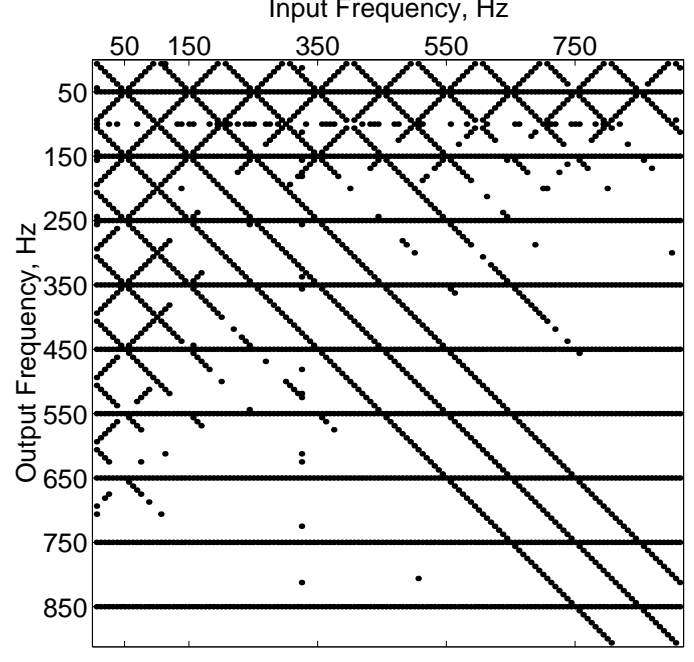

Fig. 8. Frequency coupling of single-phase rectifier.

magnitude.

\section{Three-Phase measurements}

Although common the single-phase rectifier is not the only rectifier used to convert $\mathrm{AC}$ power to $\mathrm{DC}$ power. A large amount of rectification is performed with three-phase diode rectifiers. The experimental system can be configured to make three-phase measurements. As the system has no neutral the injected perturbing current is positive or negative sequence rather than a single-phase quantity as before. In order to make measurements of three-phase quantities it is appropriate and convenient to use either sequence, orthogonal $\alpha \beta$ or rotated $d q$ components. The sequence transform resolves three wire three-phase system into two constant magnitude phasors which rotate in opposite directions. These two phasors are the positive and negative sequence of the three-phase system. In the experimental system the three-phase variables are measured and a two-phase orthogonal equivalent system formed. The two-phase system is used in the SAF controller implementation and the converter DC bus control.

A complex FFT operates on any time varying complex variable effectively performing a FFT of a locus or shape [8]. By making $\alpha$ the imaginary component of a complex signal and $-\beta$ its real component, the complex signal is of the form of (2) for a positive sequence three-phase signal and (3) for a negative sequence three-phase signal.

$$
\begin{aligned}
& -\beta+j \alpha=\cos \left(\omega_{0} t+\delta\right)+j \sin \left(\omega_{0} t+\delta\right) \\
& -\beta-j \alpha=\cos \left(\omega_{0} t-\delta\right)-j \sin \left(\omega_{0} t-\delta\right)
\end{aligned}
$$

This means that by using the orthogonal two-phase $\alpha \beta$ transform of a three-phase measurement and then taking the FFT of the complex signal formed from the two-phase representation, the sequence components of the three-phase signals can be determined. The experimental system thus performs complex sequence FFTs of the three-phase measurements. This sequence FFT is used to measure the behavior of the three-phase rectifier. 


\section{A. Three-phase rectifier measurements}

Fig. 9 shows the current waveforms for a three-phase rectifier when the current is discontinuous. The upper figure shows the base operating condition while the lower shows the load current perturbed by a $25 \mathrm{~Hz}$ positive sequence injected current. The sequence spectrum of the per-
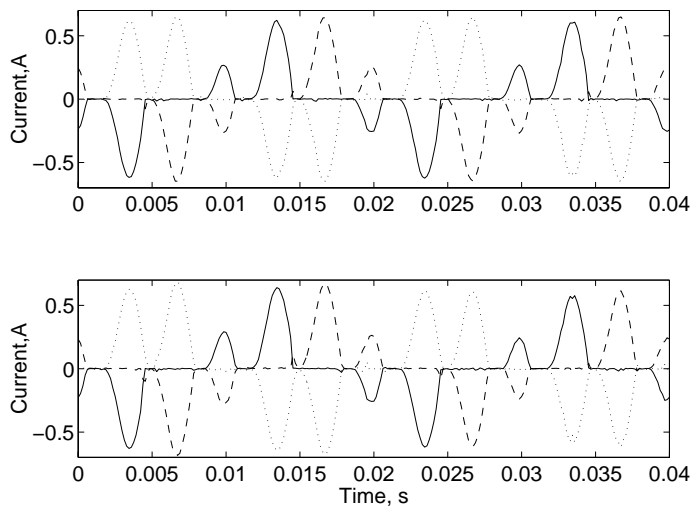

Fig. 9. Experimentally measured three-phase rectifier current with discontinuous conduction for $25 \mathrm{~Hz}$ positive sequence injected current. Notice that there is a slight difference between the upper and lower figures. Base case (upper) and perturbed (lower). (Solid line - a phase, dashed line - $\mathrm{b}$ phase, dotted line - $\mathrm{c}$ phase.)

turbed current is shown in Fig. 10 for an injected current of $25 \mathrm{~Hz}$. The base case spectrum is not removed. Note that due to the imbalance in the system voltage there are noncharacteristic harmonic currents at the negative sequence, third harmonic positive and negative sequence, fifth harmonic positive sequence and seventh harmonic negative sequence. The perturbing current's effect on the load current can be clearly seen at $25 \mathrm{~Hz}, 75 \mathrm{~Hz}, 325 \mathrm{~Hz}$ and $375 \mathrm{~Hz}$ in the positive sequence. The effect can be seen at negative sequence $275 \mathrm{~Hz}$ and negative sequence $225 \mathrm{~Hz}$. The other non-harmonic components that can be seen at negative sequence $225 \mathrm{~Hz}$ and $275 \mathrm{~Hz}$ and other such frequencies are of smaller magnitude. These are the result of the interaction of the perturbing injected current with rectifier non-characteristic harmonic behavior.

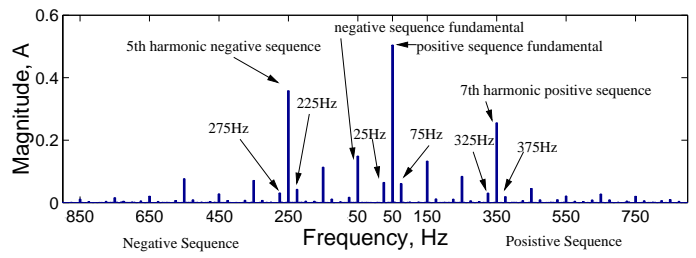

Fig. 10. Sequence spectrum of experimentally measured three-phase load current operating in discontinuous mode with injected perturbing current at $25 \mathrm{~Hz}$ positive sequence.

The sequence coupling behavior of the three-phase rectifier is shown in Fig. 11. This figure shows the frequencies at which the load response is of significant magnitude for both negative sequence perturbing current injections (shown as negative frequencies on the $\mathrm{X}$ axis in Fig. 11) and positive sequence perturbing current injections (shown as positive frequencies). Once again the base case spectrum is not removed. Different perturbation frequencies of each sequence are successively applied and the FFT of the measured load current evaluated. The dark lines in Fig. 11 show where this FFT's magnitude is larger than an arbitrarily set threshold. The way the three-phase rectifier

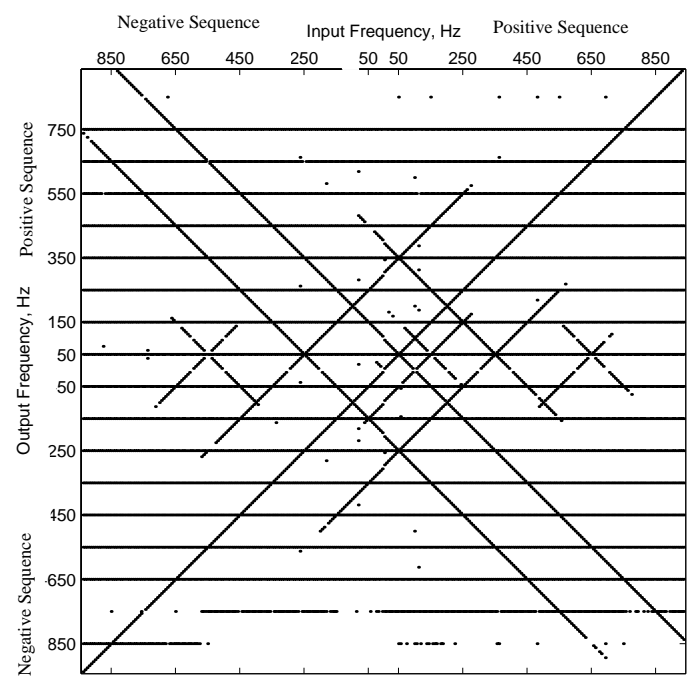

Fig. 11. Sequence coupling for three-phase rectifier. (Diagonal lines show sequence coupling, horizontal lines are base case response which has not been removed).

couples sequences can be seen by considering an input of $-50 \mathrm{~Hz}$ (negative sequence fundamental). By tracing the vertical line down from the $-50 \mathrm{~Hz}$ label it can be seen that positive sequence $450 \mathrm{~Hz}$, positive sequence $250 \mathrm{~Hz}$, positive sequence $150 \mathrm{~Hz}$, negative sequence fundamental, negative sequence $150 \mathrm{~Hz}$ and negative sequence $350 \mathrm{~Hz}$ all occur. Of considerable interest is the positive sequence third harmonic as this triple-n harmonic is not zero sequence so can propagate through a three wire three-phase system.

\section{Discussion}

The results of the measurements made using the DSP controlled power converter show that rectifier type nonlinear loads have a measurable and well patterned response when excited by a perturbing current. This means that the rectifiers are sensitive to variation in the terminal voltage caused in the measured case by the injected current. This counters the constant current source assumption made with respect to rectifier loads when they are considered connected to some power conditioner. The implications of these measurements for power conditioner connection are that since the non-linear loads are indeed sensitive to changes in their terminal voltage the behavior of the load should perhaps be considered in control system design. As the system used to make the measurements is of the type used to implement a shunt active filter this suggests that the load measurements could be made by the shunt active filter itself. This opens the door for the power conditioner to self adapt or self tune.

Perhaps of even greater interest is how the measurements show that non-linear rectifier loads have a non-zero smallsignal response and so contribute to the response of the 
total AC power system to any disturbance. The measurements made are injected current to load current change but by manipulation of equation (1) the behavior of the load can be found as a voltage to current transfer.

The frequency and sequence coupling behavior of the single-phase and three-phase rectifier respectively indicates that the behavior of both these devices is first order. That is to say that the frequencies or sequences that arise in both cases are dependent on the first multiple of the applied frequency only. These first multiple terms are typically called first order. As the size of first order effects is directly proportional to the magnitude of the applied perturbing signal the rectifier can be modelled using a constant transfer. The authors have completed such an analysis which has proved to be extremely accurate [9].

\section{A. Measurability of rectifier transfers}

Throughout the development of the experimental system, Fig. 1, to make measurements like those shown in Fig. 6 there was a doubt as to whether it is possible to actually measure the characteristics of the non-linear rectifiers. The principal concern is whether the response is sufficiently large a signal. This concern has not been found to be valid and in fact the measurements have been made with only moderate regard to the noise rejection or range of the experimental system analogue to digital converters. The use of an FFT to determine the frequency components has proved more than adequate to show that the collection of the measurements is possible.

Since the AC system plays a large part in determining the size of the current to current transfer, the relative amount of perturbation current flowing into the load decreases as the AC system impedance decreases. This can been appreciated by setting $\left[Z_{S y s}\right]$ to zero in equation (1). This does not however mean that the measurements will be impossible to make, but rather that they are zero to the precision of the measurement. When the AC system is very strong ( $\left[Z_{S y s}\right]$ very close to zero), the measured transfer is close to zero meaning that the flow of perturbing currents in the $\mathrm{AC}$ system causes very little voltage drop. When the $\mathrm{AC}$ system is relatively weak having larger impedance, the measured current to current transfer will be larger as more of the injected current will flow into the load. Therefore the ability to measure a zero transfer caused by a very strong $\mathrm{AC}$ system is no less useful than the measurement of the non-zero transfer that will occur when the $\mathrm{AC}$ system has a higher impedance.

\section{B. Relative load sensitivities}

The sensitivity of the single-phase and three-phase rectifiers have been measured. As to whether the sensitivities are larger or smaller than those of other loads cannot be determined. This requires the measurement of other load types such as induction motors and heating loads. Measurements for these types of loads can be made with the experimental system described in this paper or with any suitably modified shunt active filter.
This paper shows that the single-phase and three-phase rectifier have definite measurable responses to small signal perturbations caused by current injection into the point of connection. The single-phase rectifier has a frequency coupling characteristic while the three-phase rectifier has a sequence coupling characteristic. The sizable frequency components that occur in the rectifiers' responses to a perturbation are related only to the first multiple of the applied frequency meaning that both devices have a smallsignal behavior that is dominate by the first order terms. This means that the devices can be modelled using only first order effects.

The rectifier measurements are made with a DSP controlled IGBT power converter. Apart from extended memory and perhaps extra processing power the system is very much like the technology used in implementing shunt active filters and other power conditioners. This means that the typical shunt active filter could measure the behavior of the load and AC system to which it is connected. This may allow the adaptation of the control approach.

The measurements also give a good indication of the sensitivity of rectifier type loads and how these load contribute to power system dynamics. This is useful as it is allows the inclusion of the effects of non-linear loads in power system behavior.

\section{REFERENCES}

[1] R. Redl, P. Tenti, and J.D. van Wyk, "Power electronics polluting effect," IEEE Spectrum, vol. 34, no. 5, pp. 161-177, May 1996.

[2] H. D. Laird, S. D. Round, and R. M. Duke, "A frequency domain model of an uncontrolled single-phase voltage source rectifier," IEEE Transactions on Industrial Electronics., vol. 47, no. 3, pp. 525-532, June 2000.

[3] G. Verghese and U. Mukherji, "Extended averaging and control procedures," in Power Electronics Specialists Conference, Boulder, Colorado, USA, June 1981, pp. 329-336.

[4] H. Akagi, A. Nabae, and S. Atoh, "Control strategy of active power filters using multiple voltage source pwm converters," IEEE Transactions on Industry Applications, vol. 22, no. 3, pp. 460-465, May 1986.

[5] S. Bhattacharya, D.M. Divan, and B. Banerjee, "Synchronous frame harmonic isolator," in European Power Electronics Conference, Florence, Italy, 1991, pp. 3030-3035.

[6] H. Akagi, "Control strategy and site selection of a shunt active filter for damping of harmonic propagation in power distribution systems," IEEE Transactions on Power Delivery, vol. 12, pp. 354-363, Sept. 1997.

[7] A.R. Wood and J. Arrillaga, "Hvdc convertor waveform distortion: A frequency domain approach," IEE Proceedings on Generation, Transmission and Distribution, vol. 142, no. 1, pp. 88-96, Jan. 1995.

[8] E. O. Brigham, The Fast Fourier Transform, Prentice and Hall, Englewood Cliffs, NJ, USA, 1988.

[9] H. D. Laird, Modelling and measurements of diode rectifiers and their interaction with shunt active filters., Ph.D. thesis, Electrical and Electronic Engineering, Unversity of Canterbury, Christchurch, New Zealand, Aug. 2001. 


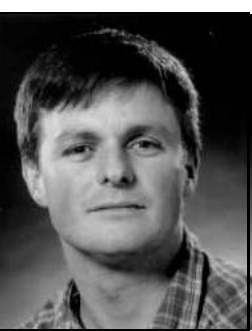

Hamish D. Laird graduated with a Bachelors (Hons) (1991) and Masters degree (1992) in Electrical and Electronic Engineering from the University of Canterbury, Christchurch, New Zealand. He then worked in New Zealand on induction motor drives and starters. He has industrial experience in the design of HVDC transmission systems and reactive power control gained in the United Kingdom. Other industrial experience includes designing and developing DC and AC drives. He completed his $\mathrm{PhD}$ in 2001 and is currently a part time lecturer at the University of Canterbury. His research and industrial interests are power electronics and electronic product development.

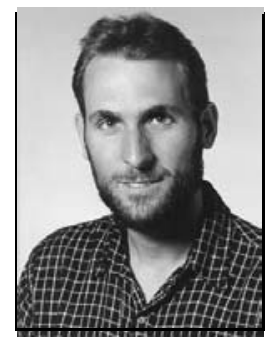

Simon D. Round (S'87-M'93) received the B.E. (Hons) and Ph.D. degrees from the University of Canterbury, Christchurch, New Zealand, in 1989 and 1993, respectively. He has held the positions of Research Associate in the Department of Electrical Engineering at the University of Minnesota and Research Fellow at the Norwegian Institute of Technology, Trondheim, Norway. Since 1995 he has been a Lecturer in the Department of Electrical and Electronic Engineering at the University of Canterbury. His current research interests are in the area of power quality and active power conditioners and electric vehicles.

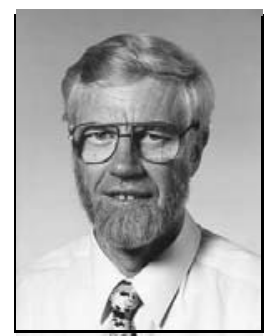

Richard M. Duke Richard M. Duke Received the B.Sc. Degree in mathematics, and the B.E. (Hons.), M.E. and Ph.D. degrees in electrical engineering from the University of Canterbury, Christchurch, New Zealand, in 1965, 1973, 1976 and 1979, respectively.He worked as an Engineer with the New Zealand Electricity Department. Since 1984 he has held a lecturing position at the University of Canterbury, where he is currently the Head of the Department of Electrical and Electronic Engineering.

He is a past Chairman of N.Z. South Section of IEEE and is a Registered Professional Engineer in N.Z. His current research interests are in the area of power quality and active filtering for power systems applications and low voltage high current switching power supplies for industrial applications. 